\title{
Efeito do envelhecimento acelerado sobre as propriedades de microconcreto reforçado com fibras longas de sisal
}

\author{
Effect of accelerated aging on the properties of \\ microconcrete reinforced with sisal fibers
}

\section{Paulo Roberto Lopes Lima \\ Romildo Dias Toledo Filho \\ Reiner Neumann \\ J oaquim Antonio Oliveira Barros}

\section{Resumo}

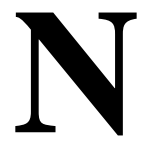

este trabalho dois microconcretos, com e sem metacaulinita, foram dosados para produção de compósitos laminados reforçados com fibras longas de sisal. Para garantir uma matriz livre de hidróxido de cálcio foi determinado o teor ideal de metacaolinita por análise termogravimétrica de pastas com teores de substituição de 20\%, 30\% e 40 nas idades de 4 h, 10 h, 24 h, 7 dias, 28 dias e 730 dias. Laminados com 30\% de metacaolinita foram submetidos a ensaio de flexão após 28 dias de cura em água e após 12 e 25 ciclos de molhagem e secagem. A análise da microestrutura foi realizada a partir de microscopia eletrônica de varredura e espectroscopia de raios $\mathrm{X}$ por energia dispersiva (EDS). Os resultados demonstram que, no microconcreto contendo apenas cimento, o hidróxido de cálcio foi lixiviado da matriz e depositado na superfície da fibra, o que resultou em ruptura brusca devido ao enfraquecimento das fibras. Com a adição de metacaolinita, a matriz mostrou-se livre de hidróxido de cálcio, e o comportamento mecânico pós-fissuração não foi alterado pelo envelhecimento. No entanto, a variação de temperatura e umidade do ciclo molhagem-secagem induziu fissuração do microconcreto, o que negativamente afetou a resistência à formação da primeira fissura do compósito e seu modo de ruptura.

Palavras-chave: Compósitos. Envelhecimento acelerado. Durabilidade.

${ }^{1}$ Paulo Roberto Lopes Lima ${ }^{1}$ Universidade Estadual de Feira de

Santana

Feira de Santana - BA - Brasil

${ }^{2}$ Romildo Dias Toledo Filho 2Universidade Federal do Rio de Rio de J aneiro - RJ - Brasil

${ }^{3}$ Reiner Neumann ${ }^{3}$ Centro de Tecnologia Mineral Rio de J aneiro - RJ - Brasil

${ }^{4} \mathrm{~J}$ oaquim Antonio Oliveira

Barros

${ }^{4}$ Universidade do Minho Guimarães - Portugal

Recebido em 15/12/16 Aceito em 21/03/18

\section{Abstract}

In this study, two microconcrete samples, with and without metakaolin, were designed for the production of laminated composites reinforced with long sisal fibres. In addition to the reference microconcrete, another microconcrete was produced by replacing cement by metakaolin. The ideal metacaolin content that would guarantee a free calcium hydroxide matrix was determined by thermogravimetric analysis of blends with $20 \%$, 30\% and $40 \%$ substitution contents at the ages of 4 h, 10 h, 24 h, 7 days, 28 days and 730 days. Laminates produced with cement substitution by $30 \%$ metakaolin were submitted to flexural test after 28 days of curing in water, and after 12 and 25 wetting and drying cycles. The analysis of the microstructure was performed by scanning electron microscopy and dispersive energy $X$-ray spectroscopy (EDS). The results demonstrate that in the microconcrete containing only cement, the calcium hydroxide was leached from the matrix and deposited on the surface of the fibre. Thus, the aged composites showed a sudden rupture due to the weakening of the fibres. With the incorporation of metakaolin, the matrix showed to be free of calcium hydroxide, and the mechanical behaviour after cracking was not altered by aging. However, the temperature and humidity variation due the wet-drying cycle has induced cracking of the microconcrete, which detrimentally affected the strength at crack initiation of these composites and their rupture mode.

Keywords: Composite. Accelerated aged. Durability.

LIMA, P. R. L.; TOLEDO FILHO, R. D.; NEUMANN, R.; BARROS, J. A. O. Efeito do envelhecimento acelerado sobre as propriedades de microconcreto reforçado com fibras longas de sisal. Ambiente Construído, Porto Alegre, v. 19, n. 1, p. 7-20, jan./ mar. 2019. 


\section{Introdução}

O uso de fibra vegetais, renováveis e com baixo consumo de energia para produção e beneficiamento, como reforço de matrizes de base cimentícia é uma alternativa tecnicamente eficiente e ecologicamente mais sustentável quando comparada com o uso de fibras manufaturadas. Compósitos reforçados com fibras de sisal podem apresentar ganho de resistência e tenacidade sob flexão após a fissuração da matriz, desde que se utilizem matrizes apropriadas (LIMA et al., 2017a).

No entanto, problemas de durabilidade foram identificados em telhas e placas corrugadas de argamassa reforçadas com fibras curtas de sisal com apenas 6 meses de uso. A investigação dos mecanismos de interação fibra-matriz identificou incompatibilidade química da matriz alcalina de cimento, que produzia nas fibras a quebra da cadeia molecular da celulose e a mineralização do lúmen da fibra, devido à migração dos hidróxidos de cálcio livre (GRAM, 1983; TOLEDO FILHO et al., 2000). A busca por soluções técnicas que aumentassem a durabilidade da fibra dentro da matriz de cimento resultou na aplicação de tratamentos da fibra (FERREIRA et al., 2015; LIMA et al., 2017b), modificações na composição da matriz de cimento, com o uso de adições minerais, e tratamento do compósito endurecido com impermeabilização ou carbonatação (TONOLI et al., 2010; GRAM, 1983; MOHR; BIERNACKI; KURTIS, 2007; LIMA; TOLEDO FILHO, 2008; CLARAMUNT et al., 2016; LIMA et al., 2017a).

A avaliação da durabilidade dos compósitos tem sido feita mediante exposição a envelhecimento natural em países de clima tropical (ROMA; MARTELLO; SAVASTANO, 2008) ou temperado (TOLEDO FILHO et al., 2003) e através de ensaios de envelhecimento acelerado, nomeadamente por meio de ciclos de molhagem e secagem, com condições variadas de tempo e temperatura (BERGSTROM; GRAM, 1984; TOLEDO FILHO et al., 2003; WEI; MEYER, 2015). No entanto, apesar de vários estudos indicarem que a adição de metacaolinita melhora a resistência mecânica e a durabilidade de matrizes à base de cimento (SILVA et al., 2014; MOTA; OLIVEIRA; CARNEIRO, 2016), o efeito da aplicação de ciclos com variação de temperatura e umidade sobre matrizes com elevado teor de adição ainda não é bem entendido.

Neste trabalho dois microconcretos reforçados com fibras longas de sisal foram produzidos utilizandose apenas cimento Portland ou um ligante composto de cimento Portland e 30\% de metacaolinita com o objetivo de avaliar o efeito do envelhecimento acelerado sobre o comportamento mecânico do compósito e sobre a resistência e composição química da matriz. O envelhecimento acelerado foi simulado através da aplicação de ciclos de molhagem por imersão em água e secagem em estufa a $70{ }^{\circ} \mathrm{C}$, tendo seu efeito sido avaliado com ensaio mecânico de flexão, microscopia eletrônica de varredura e espectroscopia de raios $\mathrm{X}$ por energia dispersiva (EDS).

\section{Método}

\section{Materiais}

A fibra de sisal (Agave sisalana) proveniente do município de Valente, Brasil, foi cortada manualmente em amostras com $400 \mathrm{~mm}$ de comprimento. A seção transversal da fibra foi avaliada por microscopia eletrônica de varredura, como mostra a Figura 1a. Verifica-se que a fibra é formada por um conjunto de microfibras (denominadas fibrocélulas) e apresenta forma irregular. A análise estatística do diâmetro da fibra de sisal, medido com microscópio óptico, demonstra que as fibras possuem grande variabilidade na dimensão, como mostra a Figura 1b, com valor médio de 0,148 mm. Ensaio de tração direta das fibras da mesma região foi realizado por Lima et al. (2014) e indicou valores de resistência à tração da ordem de $363 \mathrm{MPa}$ e módulo de elasticidade de $20 \mathrm{GPa}$.

Na produção das matrizes foi utilizado o cimento Votoran CP III - 40, com densidade de 3,00 g/ $\mathrm{cm}^{3}$ e superfície específica, determinada pelo método BET (BET multiponto, com N2), de 297,40 $\mathrm{m}^{2} / \mathrm{kg}$. A metacaolinita, com densidade de $2,57 \mathrm{~g} / \mathrm{cm}^{3}$ e superfície específica de 40.698,70 $\mathrm{m}^{2} / \mathrm{kg}$, foi produzida e caracterizada por Lima (2004). A Tabela 1 apresenta a composição química do cimento e da metacaolinita.

A areia utilizada possui módulo de finura 2,37 e massa específica de $2,64 \mathrm{~g} / \mathrm{cm}^{3}$.

\section{Misturas avaliadas}

Inicialmente um estudo foi realizado em pastas para investigar o efeito do teor de metacaolinita sobre o consumo de hidróxido de cálcio. Foram testadas três composições de aglomerantes com teores de substituição de cimento por $20 \%$, $30 \%$ e $40 \%$, em massa, de metacaolinita. As misturas foram moldadas em sacos plásticos, que foram vedados após introdução de cimento e água (fator águaaglomerante de 0,4) para evitar a carbonatação das amostras. Após a homogeneização das pastas com amassamento manual, elas foram mantidas nos sacos plásticos até a idade do ensaio. A evolução da 
hidratação foi avaliada em diferentes idades: 4 h, 10 h, 24 h, 7 dias, 28 dias e 730 dias.

Os compósitos laminados foram produzidos com dois tipos de matrizes (M1 e M2) com traço, em massa, de 1:1 (aglomerante:areia) com fator águaaglomerante de 0,3 e adição de 0,5\%, em massa, de superplastificante à base de naftaleno sulfonado. A matriz M1 contém apenas cimento, e a matriz M2 utiliza 30\%, em massa, de metacaolinita em substituição de cimento.

\section{Produção dos compósitos laminados}

Para a produção dos laminados, os materiais foram lançados em moldes metálicos com dimensões internas de $400 \mathrm{~mm}$ x $400 \mathrm{~mm}$. O reforço principal foi dosado para um volume de fibras longas de cerca de $3 \%$, distribuído em duas camadas com 1,5\% de fibra em cada uma. A espessura média dos laminados foi de $15 \mathrm{~mm}$.

Inicialmente foi lançada uma camada de microconcreto de $5 \mathrm{~mm}$, distribuída uniformemente no fundo do molde. As fibras foram lançadas com comprimento de $400 \mathrm{~mm}$ sobre o microconcreto, sendo distribuídas de forma alinhada com a ajuda de um rolo plástico, como mostra a Figura 2a. Após o lançamento da última camada de microconcreto, os corpos de prova foram submetidos a pressão de moldagem de 2 MPa (Figura 2b) em uma prensa Shimadzu de $100 \mathrm{kN}$, tendo sido observadas a expulsão de água e a redução da espessura da placa no estado fresco.

Após 24 h, os laminados foram desmoldados e colocados em água até a idade de 28 dias em um tanque de cura à temperatura de $25 \pm 1{ }^{\circ} \mathrm{C}$. Os compósitos foram denominados como C-M1, quando produzido com a matriz M1, e C-M2, quando a matriz M2 com metacaolinita foi utilizada.

\section{Métodos de ensaio}

Para a avaliação do teor de hidróxido de cálcio livre nas pastas foram realizadas análises térmicas em um equipamento da TA Instrument, modelo SDT Q600, com taxa de aquecimento de $10{ }^{\circ} \mathrm{C} / \mathrm{min}$, de 35 a $1.000{ }^{\circ} \mathrm{C}$, utilizando $100 \mathrm{~mL} / \mathrm{min}$ de fluxo de nitrogênio. Inicialmente as amostras foram secas dentro do equipamento a uma taxa de $1{ }^{\circ} \mathrm{C} / \mathrm{min}$ de 30 a $35^{\circ} \mathrm{C}$, seguido de uma secagem isotérmica a $35^{\circ} \mathrm{C}$ por $1 \mathrm{~h}$ para eliminar toda a água livre.

\section{Figura 1 - Fibra de sisal}

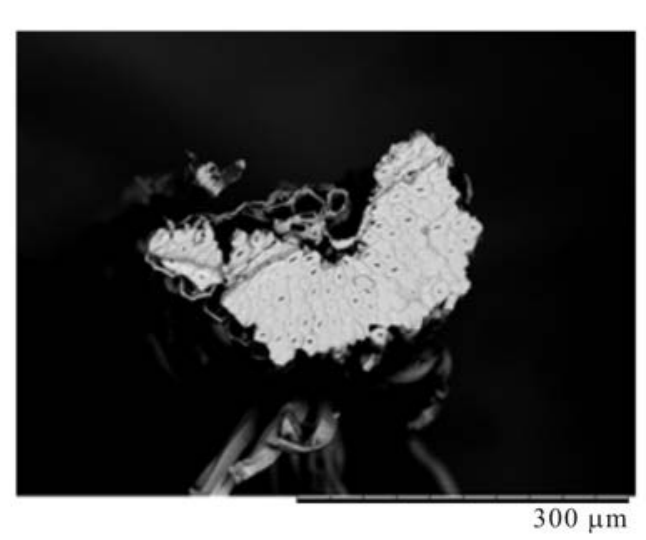

(a) Micrografia da seção transversal

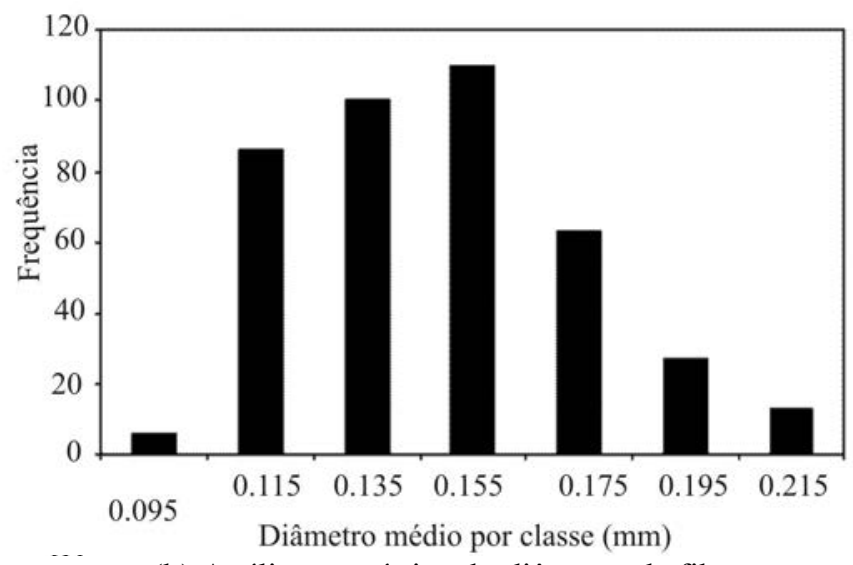

(b) Análise estatística do diâmetro da fibra

Tabela 1 - Composição química (\% massa) do cimento e metacaolinita

\begin{tabular}{c|c|c}
\hline Composto & Cimento & Metacaolinita \\
\hline $\mathrm{SiO}_{2}$ & 24,94 & 52,46 \\
$\mathrm{CaO}$ & 53,05 & - \\
$\mathrm{Al}_{2} \mathrm{O}_{3}$ & 7,50 & 44,24 \\
$\mathrm{TiO}_{2}$ & & 1,28 \\
$\mathrm{Fe}_{2} \mathrm{O}_{3}$ & 2,62 & 2,06 \\
$\mathrm{MgO}$ & 0,85 & - \\
$\mathrm{Na}_{2} \mathrm{O}$ & 0,12 & - \\
\hline
\end{tabular}


Figura 2 - Processo de produção dos laminados

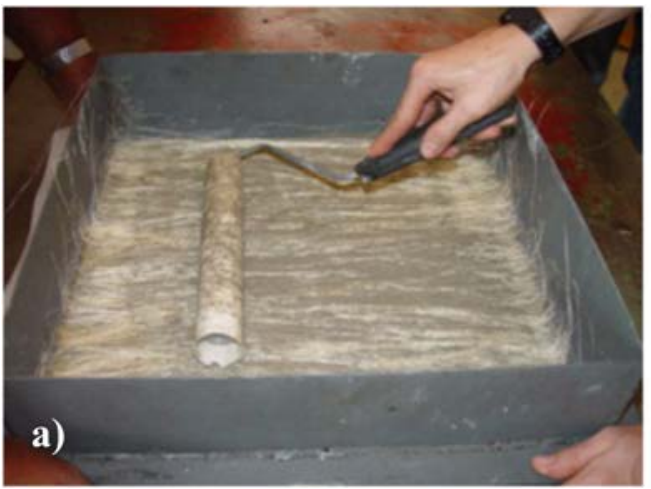

(a) Lançamento da camada de fibra

Para a realização do ensaio de flexão os compósitos foram cortados em corpos de prova com dimensão $100 \mathrm{~mm}$ x $400 \mathrm{~mm}$. O ensaio de flexão foi realizado numa máquina universal Shimadzu com célula de carga de $20 \mathrm{kN}$, tendo-se adotado uma configuração de quatro pontos de carga, vão interno de $300 \mathrm{~mm}$ e taxa de deslocamento de $0,5 \mathrm{~mm} / \mathrm{min}$. A partir da curva carga-deslocamento foram calculados:

(a) resistência ao início da fissuração da matriz (FCS), dada por Eq. 1:

$\mathrm{FCS}=\mathrm{P}_{\mathrm{f}} \mathrm{L} / \mathrm{be}^{2}$

Eq. 1

Onde $\mathrm{L}(=300 \mathrm{~mm})$, e $(=10 \mathrm{~mm})$ e b $(=40 \mathrm{~mm})$ representam respectivamente o vão interno, a a espessura e a largura do corpo de prova. Neste trabalho, a carga $\mathrm{P}_{\mathrm{f}}$ corresponde à máxima carga antes do surgimento da primeira fissura e identificada pela primeira queda de carregamento identificada na curva carga-deslocamento.

(b) máxima tensão pós-fissuração $\left(\sigma_{b}\right)$, determinada a partir da carga máxima $\left(\mathrm{P}_{\max }\right)$ resistida pelo compósito após a primeira fissura, também obtida através da Eq. (1) pela substituição de $\mathrm{P}_{\mathrm{f}}$ por $\mathrm{P}_{\max }$;

(c) fator de carga $\mathrm{P}^{*}=\mathrm{P}_{\mathrm{n}} / \mathrm{P}_{\mathrm{f}}$, definido pela norma NBN B 15238 (INSTITUTE..., 1992), onde $\mathrm{P}_{\mathrm{f}}$ é a carga de início da fissuração e $\mathrm{P}_{\mathrm{n}}$ é a carga para cada valor de deslocamento n, adotado como 0,5 mm, 0,75 mm, $1 \mathrm{~mm}, 2 \mathrm{~mm}, 3 \mathrm{~mm}, 6 \mathrm{~mm}$ e 12 $\mathrm{mm}$;

(d) tenacidade $\left(\mathrm{T}_{\mathrm{JCI}}\right)$, definida como a energia necessária para fletir o compósito laminado até uma deflexão no meio de vão de L/150, isto é, 2,00 $\mathrm{mm}$ no presente estudo.

Para o envelhecimento acelerado foi definido o ciclo de 48 h, sendo $17 \mathrm{~h}$ em água a $22^{\circ} \mathrm{C}$, $30 \mathrm{~h}$ em estufa a $70{ }^{\circ} \mathrm{C}$ e $1 \mathrm{~h}$ em condições ambientais naturais, antes da próxima saturação. Após o envelhecimento foram realizados ensaios de flexão nos laminados e microscopia nas fibras e matriz.

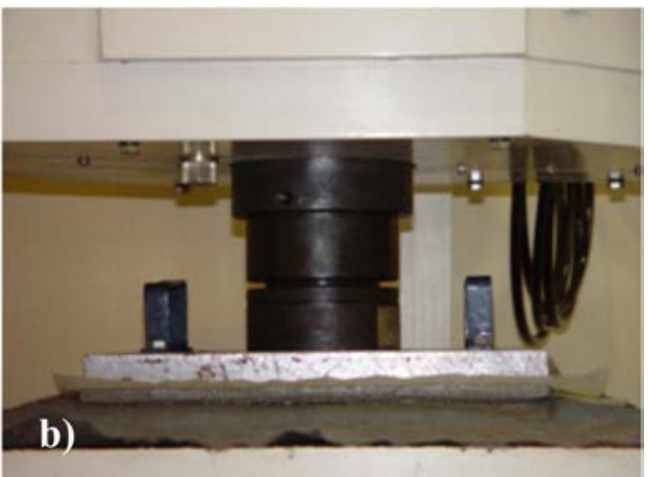

(b) Moldagem sob pressão

Análises de microscopia de varredura (MEV) foram executadas num equipamento FEI Quanta 400 usando imagem de elétrons retroespalhados e espectroscopia de raios $\mathrm{X}$ por energia dispersiva (EDS) com sistema Bruker Nano Quantax 800 e detector XFlash 4030, realizadas em amostras embutidas a frio em resina epóxi lixadas sobre discos de diamante em matriz metálica de 40, 15, 9 e $6 \mu \mathrm{m}$, polidas com suspensão diamantada em base aquosa de 3 e $1 \mu \mathrm{m}$ sobre panos sintéticos muito duros, e cobertas com carbono.

\section{Resultados e discussão}

\section{Avaliação do teor de hidróxido de cálcio}

A utilização de fibras vegetais no reforço de elementos construtivos com matrizes convencionais, como concretos e argamassas, resultou em materiais com resistência mecânica adequada, mas com baixa durabilidade (GRAM, 1983). A investigação da interação química entre a fibra e a matriz demonstrou que um dos principais mecanismos de deterioração da fibra vegetal é a mineralização das fibras pela migração do hidróxido de cálcio da matriz de cimento para o interior do lúmen da fibra durante processos de molhagem e secagem dos produtos (MOHR; BIERNACKI; KURTIS, 2007). A formação do hidróxido de cálcio é, de maneira simplificada, expressa pelas seguintes reações de hidratação do cimento Portland (Equações 2 e 3):

$$
\begin{aligned}
& 2 \mathrm{C}_{3} \mathrm{~S}+6 \mathrm{H} \rightarrow \mathrm{C}_{3} \mathrm{~S}_{2} \mathrm{H}_{3}+3 \mathrm{CH} \\
& 2 \mathrm{C}_{2} \mathrm{~S}+4 \mathrm{H} \rightarrow \mathrm{C}_{3} \mathrm{~S}_{2} \mathrm{H}_{3}+\mathrm{CH}
\end{aligned}
$$

Onde:

$$
\begin{aligned}
& \mathrm{C}=\mathrm{CaO} ; \\
& \mathrm{S}=\mathrm{SiO}_{2} ; \\
& \mathrm{H}=\mathrm{H}_{2} \mathrm{O} ; \mathrm{e}
\end{aligned}
$$


$\bar{S}=\mathrm{SO}_{3}$.

O processo de degradação química da fibra tem sido minimizado pelo uso de adições pozolânicas que consomem o hidróxido de cálcio $(\mathrm{CH})$ gerado nas reações de hidratação do cimento e formam novos produtos de hidratação (PAPADAKIS; FARDIS; VAYENAS, 1992):

$$
\begin{array}{lr}
2 \mathrm{~S}+3 \mathrm{CH} \rightarrow \mathrm{C}_{3} \mathrm{~S}_{2} \mathrm{H}_{3} & \text { Eq. } 4 \\
A+\mathrm{F}+8 \mathrm{CH}+18 \mathrm{H} \rightarrow \mathrm{C}_{8} A F H_{26} & \text { Eq. } 5 \\
A+C \bar{S} H_{2}+3 \mathrm{CH}+7 \mathrm{H} \rightarrow \mathrm{C}_{4} A \bar{S} H_{12} & \text { Eq. } 6 \\
A+4 C H+9 H \rightarrow C_{4} A H_{13} & \text { Eq. } 7
\end{array}
$$

Onde:

$\mathrm{A}=\mathrm{Al}_{2} \mathrm{O}_{3} ; \mathrm{e}$

$\mathrm{F}=\mathrm{Fe}_{2} \mathrm{O}_{3}$.

A eficiência do uso de pozolanas na redução da deterioração das fibras vegetais em compósitos à base de cimento, no entanto, é influenciada pelo tipo de pozolana e pela proporção de substituição em relação à massa de cimento (LIMA et al., 2017a), visto que a composição química, a finura e a cristalinidade das pozolanas afetam sua reatividade com o hidróxido de cálcio. Por isso, neste trabalho foram avaliados teores de substituição de 20\%, 30\% e $40 \%$ de metacaolinita. O efeito de cada teor de adição sobre o consumo de hidróxido de cálcio foi avaliado com o uso de técnicas de análise térmica e avaliação das curvas de termogravimetria (TG), de termogravimetria derivada (DTG) e diferencial (DTA) para diferentes tempos de hidratação, como mostra a Figura 3.

A Figura 3a mostra as curvas obtidas na análise térmica de uma pasta de cimento Portland submetida à variação de temperatura de 35 a 1.000 ${ }^{\circ} \mathrm{C}$. Verifica-se na curva DTG a presença de quatro picos característicos (DWECK et al., 2000): entre 50 e $200{ }^{\circ} \mathrm{C}$ os picos correspondem à água liberada das fases $\mathrm{CSH}$ (tobermorita) e etringita; entre $380 \mathrm{e}$ $450{ }^{\circ} \mathrm{C}$ há um pico correspondente à água perdida pela desidratação do hidróxido de cálcio; e entre 600 e $700{ }^{\circ} \mathrm{C}$ é verificado um pico correspondente à perda de $\mathrm{CO}_{2}$ devido à decomposição do carbonato de cálcio $\left(\mathrm{CaCO}_{3}\right)$. Na curva DTA é possível verificar o pico endotérmico entre 380 e $450{ }^{\circ} \mathrm{C}$, que indica a ocorrência da reação de desidratação do hidróxido de cálcio. A partir da perda de massa ocorrida entre essas temperaturas é possível calcular o teor de hidróxido de cálcio da mistura (LIMA; TOLEDO FILHO, 2008). Analisando o resultado da análise térmica das pastas com substituição de $40 \%$ de cimento por metacaolinita, mostrada na Figura 3b, verifica-se que, apesar da identificação das reações associadas à decomposição da tobermorita, etringita e carbonato de cálcio, não se verificam picos associados à desidratação do hidróxido de cálcio, o que confirma que este foi consumido nas reações de hidratação (Eq. 4 a 7), em que se geraram novos compostos hidratados.

A avaliação do processo de hidratação das pastas de cimento e cimento com $40 \%$ de metacaolinita, em diferentes idades, foi realizada através das curvas de DTG mostradas nas Figuras 4 e 5. Para a pasta de cimento, o surgimento do pico de desidratação do hidróxido de cálcio é observado a partir da décima hora de hidratação até a idade de 730 dias. As perdas de água corresponderam a $0,75 \%, 1,50 \%$, $1,76 \%$ e $1,93 \%$ da massa da amostra a $36{ }^{\circ} \mathrm{C}$ para as idades de $10 \mathrm{~h}, 24 \mathrm{~h}, 7$ dias e 28 dias respectivamente. Isso corresponde a uma desidroxilação de 3,08\%, 6,17\%, 7,24\% e 7,90\% de hidróxido de cálcio respectivamente. Para pastas com $40 \%$ de metacaolinita não é identificada a formação do hidróxido de cálcio, mesmo após 730 dias de hidratação.

\section{Figura 3 - Análise termogravimétrica (TG), termogravimetria derivada (DTG) e diferencial (DTA) de a) pastas de cimento e b) pastas com $40 \%$ de metacaolinita}
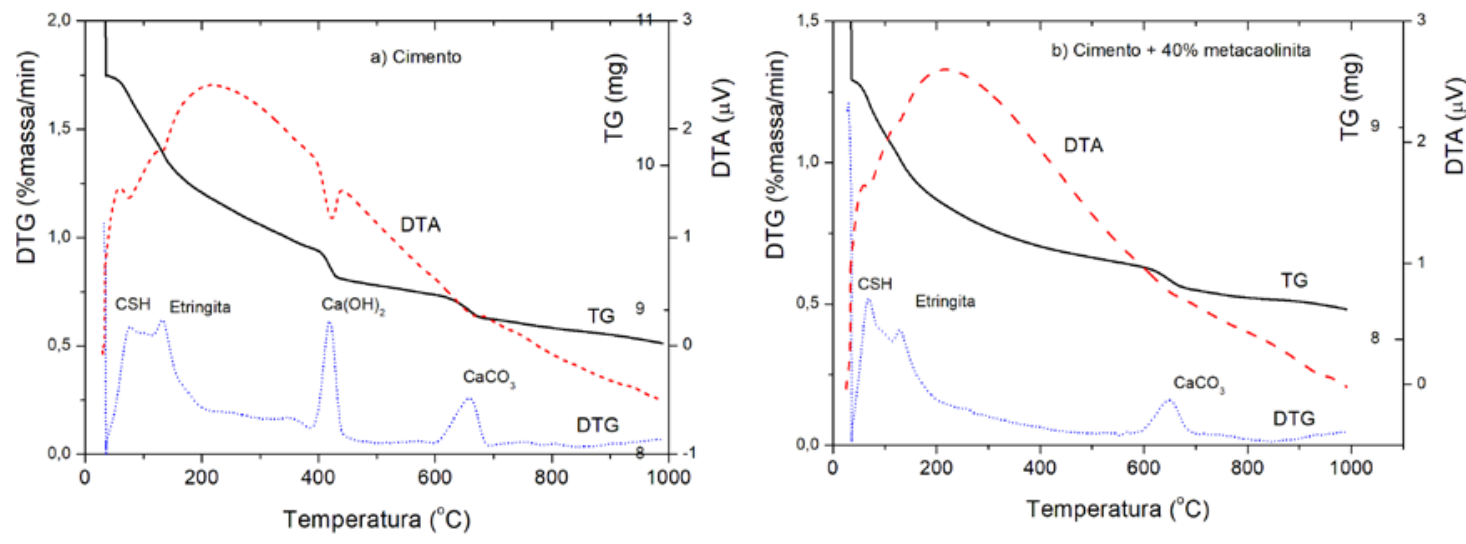
Figura 4 - Análise termogravimétrica diferencial (DTG) de pastas de cimento para várias idades
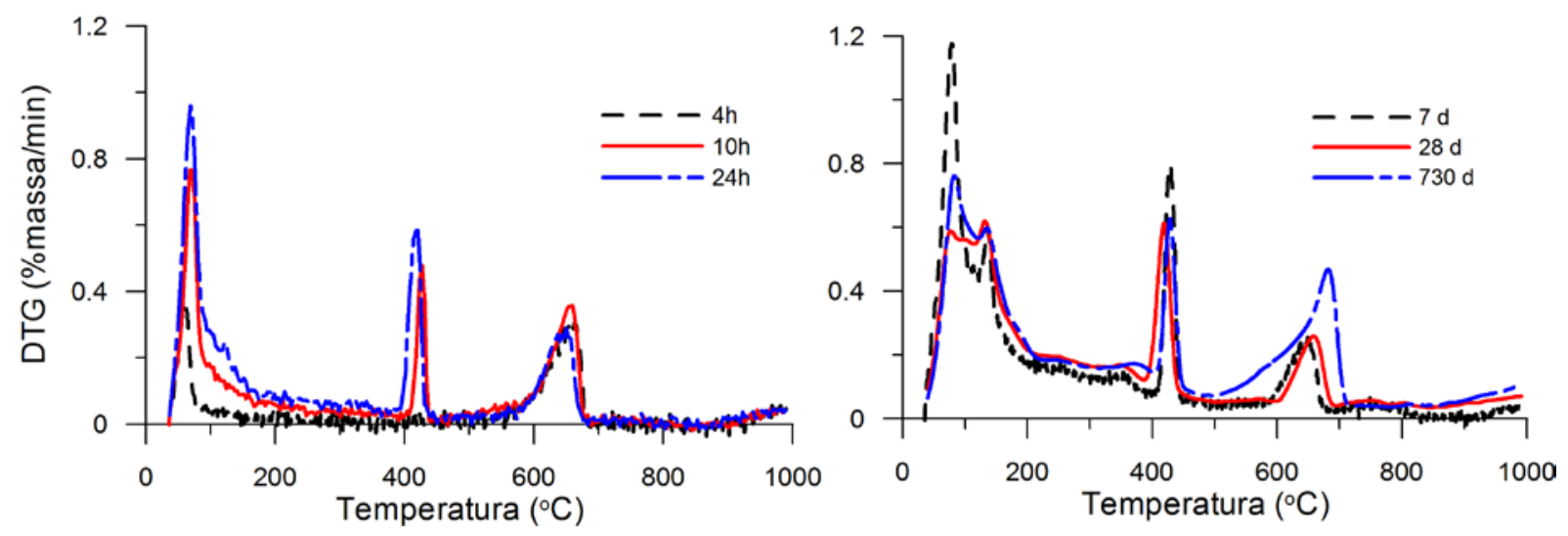

Figura 5 - Análise termogravimétrica diferencial (DTG) de pastas de cimento com $\mathbf{4 0} \%$ de metacaolinita para várias idades
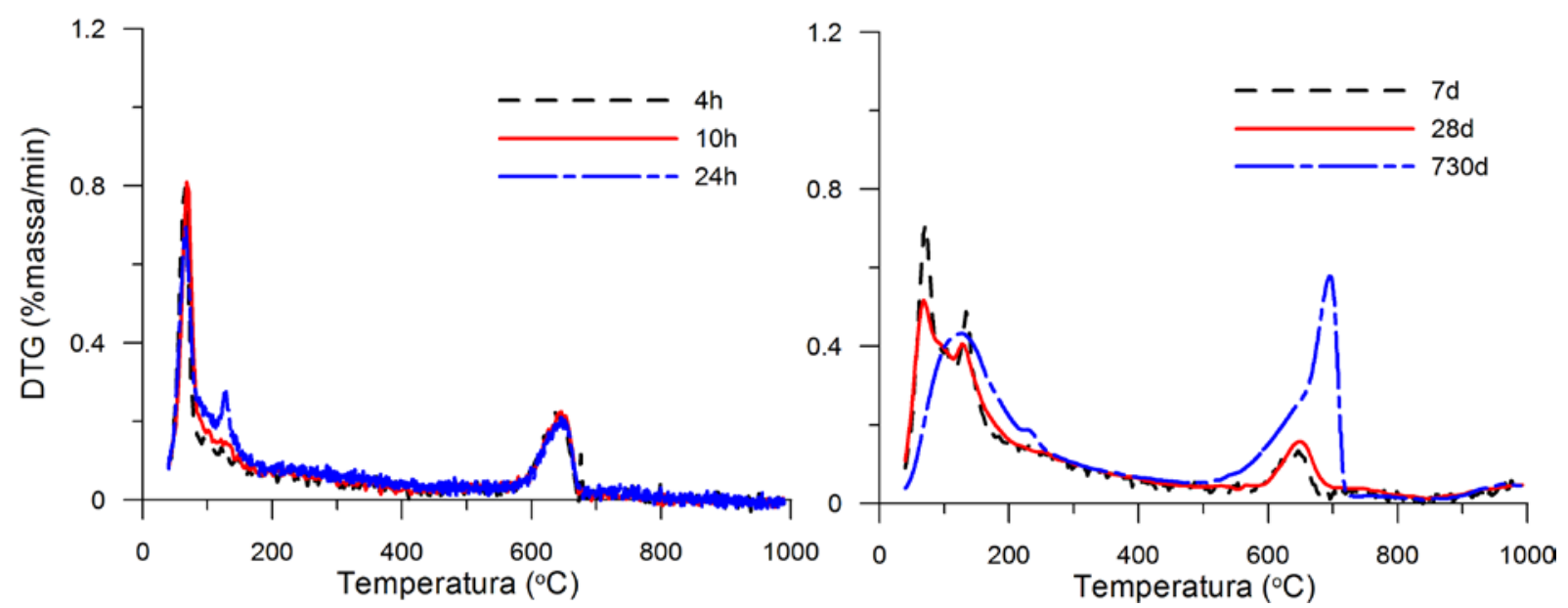

Figura 6 - Análise termogravimétrica diferencial (DTG) de pastas de cimento com $20 \%$ e $30 \%$ de metacaolinita

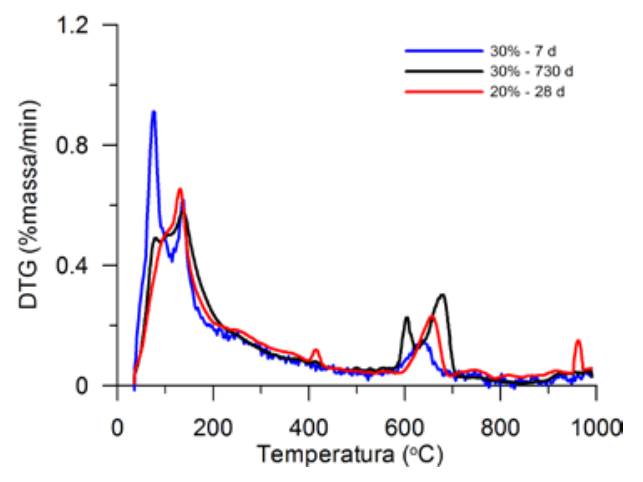

Pastas com 20\% e 30\% de adição de metacaolinita foram também avaliadas, como mostra a Figura 6, e os valores de teores de hidróxido de cálcio livres calculados estão apresentados na Tabela 2 . Verifica-se que matrizes livres de hidróxido de cálcio foram conseguidas com substituição de 30\% e $40 \%$ do cimento (CP) por metacaolinita (MK) devido às reações pozolânicas descritas nas Equações 3 a 6. 
Tabela 2 - Teores de hidróxido de cálcio nas pastas mistas em várias idades obtidos pela análise termogravimétrica

\begin{tabular}{c|c|c|c|c|c|c}
\hline $\begin{array}{c}\text { Proporção das pastas (em massa) } \\
\text { Aglomerante (cimento + adição): } \mathbf{A} / \mathbf{C}\end{array}$ & $\mathbf{4} \mathbf{h}$ & $\mathbf{1 0} \mathbf{~ h}$ & $\mathbf{2 4} \mathbf{~ h}$ & $\mathbf{7}$ dias & $\mathbf{2 8}$ dias & $\mathbf{7 3 0}$ dias \\
\hline 1,0 CP : 0,4 & 0,00 & 3,08 & 6,17 & 7,24 & 7,90 & 6,67 \\
$(0,8 \mathrm{CP}+0,2 \mathrm{MK}): 0,4$ & & - & - & - & 0,82 & - \\
$(0,7 \mathrm{CP}+0,3 \mathrm{MK}): 0,4$ & & - & - & 0,00 & - & 0,00 \\
$(0,6 \mathrm{CP}+0,4 \mathrm{MK}): 0,4$ & 0,00 & 0,00 & 0,00 & 0,00 & 0,00 & 0,00 \\
\hline
\end{tabular}

\section{Comportamento mecânico}

Na Figura 7 são apresentadas as curvas tensão versus deslocamento nos compósitos com matriz de cimento (C-M1) e com matriz com metacaolinita (C-M2) após cura em água (0 ciclos) e após serem submetidas a 12 ciclos e 25 ciclos de envelhecimento acelerado. Valores médios de FCS, $\sigma_{b}$ e de tenacidade $T_{J C I}$ dos compósitos, com os respectivos coeficientes de variação $(\mathrm{CV})$, são apresentados na Tabela 3.

\section{Fissuração da matriz}

Avaliando a resistência de início de fissuração do compósito (FCS) sem envelhecimento, verifica-se que a adição de metacaolinita resultou em uma redução da ordem de $49,6 \%$. Usando $10 \%$ e $30 \%$ de metacaolinita, Mohr, Biernacki e Kurtis (2007) também observaram que os compósitos exibiram 6,7-24,3\% também aos 28 dias de hidratação, menor FCS que a do compósito de controle, sem adição. Lima e Toledo Filho (2008) verificaram que a adição de $30 \%$ de metacaolinita reduziu em cerca de $36 \%$ o valor de FCS para misturas sem envelhecimento. A inclusão de metacaolinita como substituição parcial do cimento aumenta a resistência mecânica do concreto até determinado teor, denominado nível de substituição ótima, que pode variar de 15\% (DINAKAR; SAHOO; SRIRAM, 2013) a 20\% (WILD; KHATIB; JONES, 1996), após o qual a resistência mecânica começa a se reduzir. De acordo com Dinakar, Sahoo e Sriram (2013), essa redução pode ser explicada pelo efeito de diluição do clínquer, que é um resultado da substituição de uma parte do cimento pela quantidade equivalente de metacaolinita. Isso porque, para que haja a continuidade das reações pozolânicas, mostradas nas Equações 4 a 7, é preciso que haja hidróxido de cálcio $(\mathrm{CH})$ suficiente na mistura. Quando todo o $\mathrm{CH}$ é consumido, a metacaolinita em excesso não tem com quem reagir quimicamente e fica como inerte na mistura, podendo gerar o efeito filler. Analisando os resultados apresentados por Courard et al. (2003), verifica-se aumento de resistência à flexão até um teor ótimo de $5 \%$, com redução gradual para $10 \%$ e 15\% de substituição até se atingir uma resistência similar à do concreto de referência para teor de metacaolinita de $20 \%$.

No presente trabalho, em que os compósitos foram produzidos sob pressão, a redução de FCS com a adição de metacaolinita pode também estar associada a uma relação água-aglomerante efetiva maior. Apesar de possuírem a mesma relação águaaglomerante inicial, durante a prensagem para a produção dos laminados, observou-se visualmente menor expulsão de água da mistura M2 em relação à mistura M1 (sem pozolana).

Após o envelhecimento acelerado, verifica-se redução de FCS para ambos os compósitos: C-M1 apresentou redução de $8,4 \%$ e $9,4 \%$ após ser submetido a 12 ciclos e 25 ciclos de envelhecimento respectivamente; e C-M2 apresentou perdas maiores de resistência, quantitativamente 36,7\% e $36,9 \%$ para 12 e 25 ciclos, respectivamente, acompanhando a tendência de redução apontada pela literatura para compósitos com matriz contendo metacaolinita submetidos a grande variações de temperatura, como mostra a Figura 8.

A inspeção da superfície dos compósitos durante os ciclos de envelhecimento acelerado permitiu verificar que as placas com metacaolinita apresentavam dois tipos de danos:

(a) intensa microfissuração gerada pelos ciclos de choque térmico (Figura 9a); e

(b) fissuras transversais, na face superior e na face inferior, e longitudinais, na lateral, ao longo da camada de fibra, mostradas a Figura 9b, geradas pelo gradiente de secagem da superfície em relação ao ponto do corpo de prova onde estavam localizadas as fibras.

As fissuras laterais surgiram possivelmente na interface fibra-matriz devido à grande diferença de coeficiente de dilatação térmica entre a camada de interface e a fibra. Assim, as tensões de tração que estão na base das fendas observadas durante os ciclos de secagem-molhagem acompanhados de variação de temperatura terão sido devidas à restrição que as fibras ofereceram à deformação da matriz envolvente. A água armazenada nas fibras terá também contribuído para o desenvolvimento de tensões na zona de interface entre as fibras e a matriz envolvente devido a sua conversão em vapor 
quando o laminado foi sujeito a aumento de temperatura no processo de secagem, bem como à variação dimensional das fibras devido a essa mudança de estado. Esse processo poderá ter tido evolução não uniforme na espessura do laminado, tendo gerado uma curvatura que justifica a ocorrência de fissuras transversais (Figura 9b).

Figura 7 - Curvas típicas tensão versus deslocamento a meio vão de compósitos com (a) matriz de cimento e (b) $30 \%$ de cimento substituído por metacaolinita

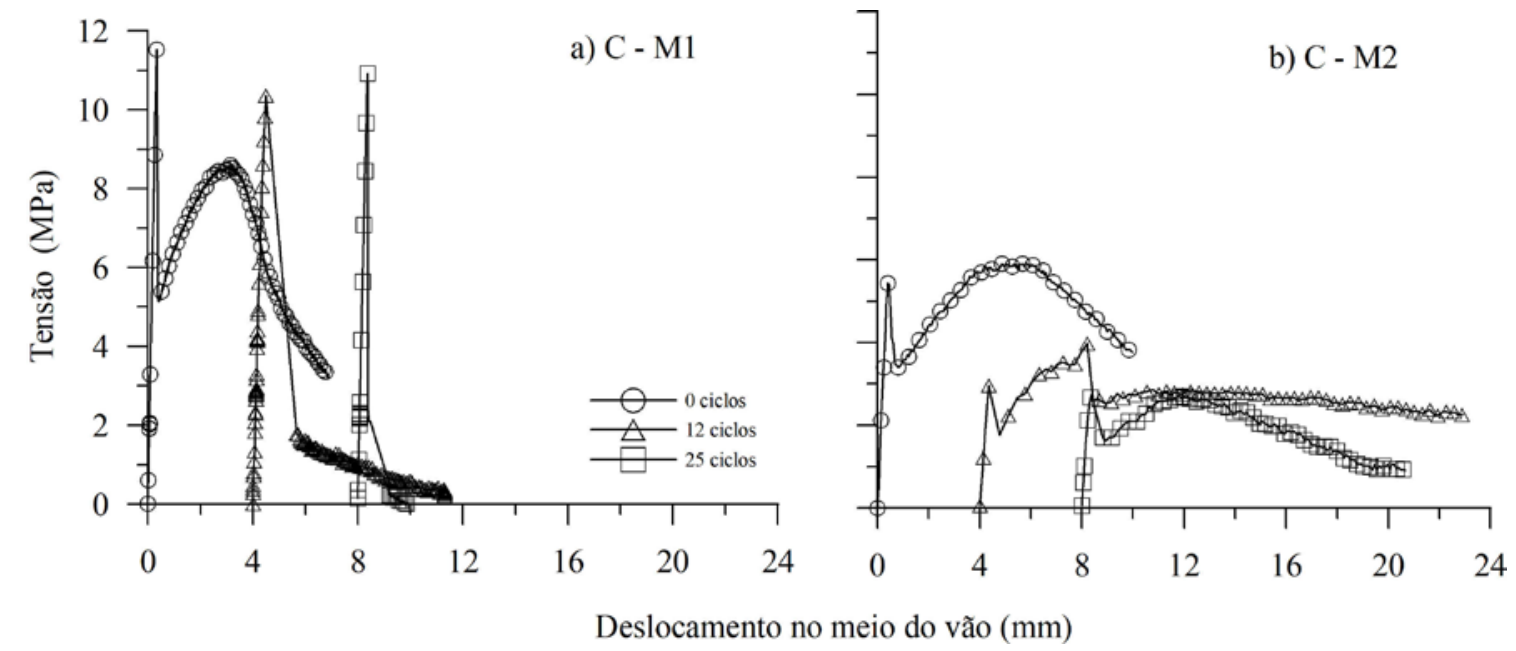

Tabela 3 - Resultados experimentais do ensaio de flexão

\begin{tabular}{c|c|c|c}
\hline $\begin{array}{c}\text { Compósito } \\
\text { laminado }\end{array}$ & Ciclos & $\begin{array}{c}\text { FCS (CV) } \\
\mathbf{( M P a ) ( \% )}\end{array}$ & $\begin{array}{c}\sigma_{\mathbf{b}}(\mathbf{C V}) \\
\mathbf{( M P a ) ( \% )}\end{array}$ \\
\hline \multirow{3}{*}{ C - M1 } & 0 & $10,88(8,3)$ & $8,40(2,15)$ \\
& 12 & $9,97(3,4)$ & - \\
\hline & 25 & $9,85(7,8)$ & - \\
C - M2 & 0 & $5,48(2,4)$ & $6,27(4,5)$ \\
& 12 & $3,47(1,5)$ & $3,66(8,9)$ \\
\hline
\end{tabular}

Figura 8 - Efeito do envelhecimento sobre a resistência de início de fissuração da matriz (FCS) de compósitos com metacaolinita

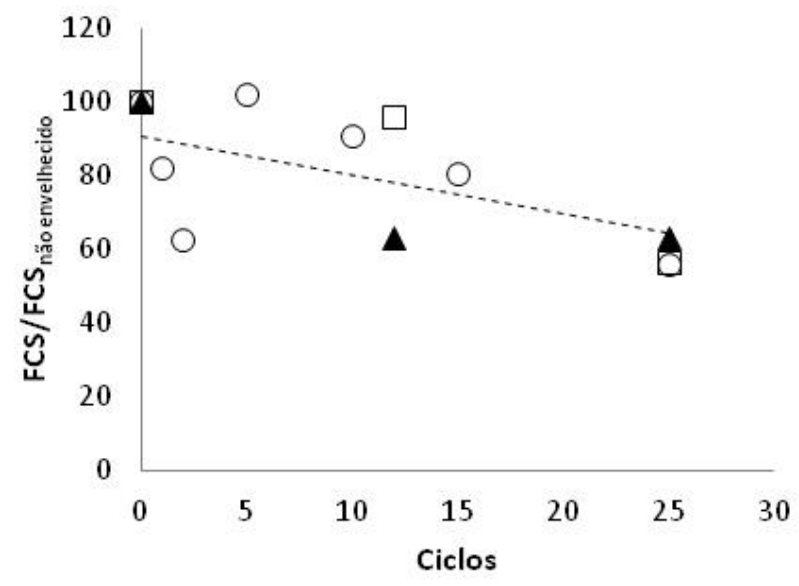

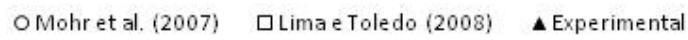


Figura 9 - Efeito do envelhecimento sobre a matriz com metacaolinita: (a) microfissuração; e (b) fissuras laterais e longitudinais

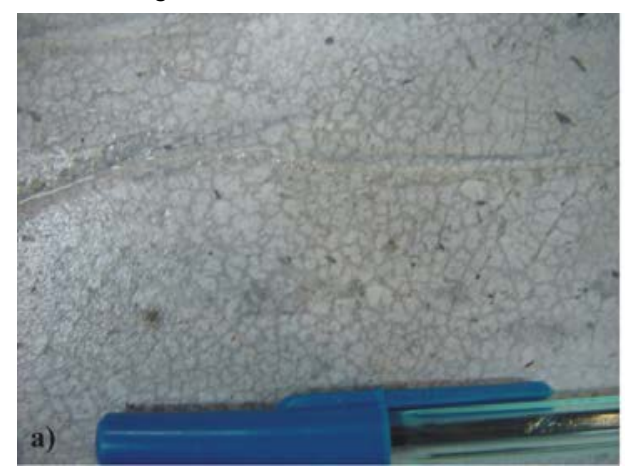

\section{Comportamento pós-fissuração do compósito}

Nos compósitos, após a formação da primeira fissura, a capacidade de carga que a matriz suporta é transferida para as fibras que a atravessam, passando-se a mobilizar os mecanismos de reforço das fibras. Apesar da utilização de fibras longas neste trabalho, a relativa baixa aderência das fibras vegetais com matrizes de base cimentícia contribuiu para a ocorrência de queda de capacidade de carga do laminado imediatamente após a fissuração da matriz, como mostra a Figura 7. Com o processo de tensionamento das fibras, verifica-se então uma fase de ganho de resistência, até um valor máximo $\sigma_{b}$. Após esse ponto, a ligação fibra-matriz entra numa fase de progressivo e intenso dano, justificando a fase de amolecimento estrutural verificado nos ensaios dos laminados. A ruptura do laminado acontece então pelo arrancamento gradual das fibras, pelo que as condições de ligação entre as fibras e a matriz são determinantes para a capacidade do laminado, tal como demonstrado em Barros, Silva e Toledo Filho (2016).

Os laminados C-M1 e C-M2 apresentaram aos 28 dias tensões máximas pós-fissuração, $\sigma_{b}$, iguais a 8,4 MPa e 6,3 MPa respectivamente. Comparandose as tensões de fissuração (FCS), verifica-se que o laminado C-M1 apresenta redução na capacidade resistente da ordem de $77 \%$. Para o laminado com metacaolinita, por outro lado, a tensão pósfissuração é $114 \%$ maior que FCS.

$\mathrm{O}$ efeito do envelhecimento altera significativamente o comportamento pós-fissuração do laminado C-M1, que passa a ter redução significativa na capacidade de manutenção de carga. O valor de $\sigma_{b}$ se reduz a zero. Avaliando o fator de carga, $\mathrm{P}^{*}$, do laminado C-M1, verifica-se que o surgimento da primeira fissura é seguido da perda total de resistência após 12 e 25 ciclos, como mostra a Figura 10. Esse comportamento está

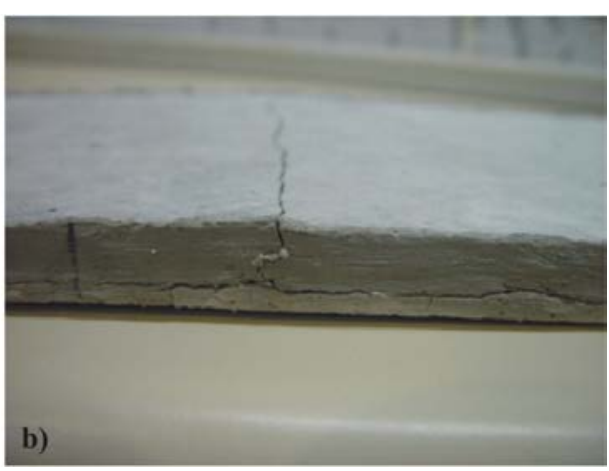

diretamente associado à perda de resistência da fibra de sisal, visto que os ciclos molhagemsecagem aceleram o processo de migração do hidróxido de cálcio para o interior da fibra e causam a degradação química.

Para o laminado C-M2, por outro lado, o valor de $\sigma_{b}$ reduziu $41,6 \%$ e $52,1 \%$ após 12 e 25 ciclos de envelhecimento, respectivamente, com relação ao laminado sem envelhecimento. De acordo com Mohr, Biernacki e Kurtis (2007), a máxima tensão pós-fissuração compósitos com 30\% de metacaolinita pode apresentar redução até 43,5\% após a aplicação de 25 ciclos molhagem-secagem.

Enquanto para o laminado C-M1 há redução significativa de $\mathrm{P}^{*}$ após 12 e 25 ciclos de envelhecimento, para o laminado C-M2 o comportamento de $\mathrm{P}^{*}$ é praticamente o mesmo para todas as situações de envelhecimento. Isso demonstra que a fibra de sisal não sofreu degradação importante, visto que continua a transferir tensões entre as fissuras.

Para os compósitos C-M2, com metacaolinita, o processo de ruptura foi precedido de uma delaminação e deslizamento entre camadas de matrizes, que foram microfissuradas pelo possível choque térmico, como mostram as Figuras 11a e 11b. Isso indica também que houve fissuração na interface fibra-matriz durante o processo de envelhecimento acelerado.

Após o ensaio de flexão, a inspeção visual revelou ocorrência de rotura das fibras rente à a superfície de fratura do compósito C-M1, como mostra a Figura 11c, o que indica que o comprimento de arrancamento das fibras foi severamente afetado pelo envelhecimento acelerado. Para o laminado CM2, o comprimento de arrancamento da fibra foi similar ao do compósito sem envelhecimento, o que indica que a matriz livre de hidróxido de cálcio foi capaz de manter a integridade da fibra de sisal apesar dos ciclos de envelhecimento 
Figura 10 - Efeito do envelhecimento sobre o fator de carga

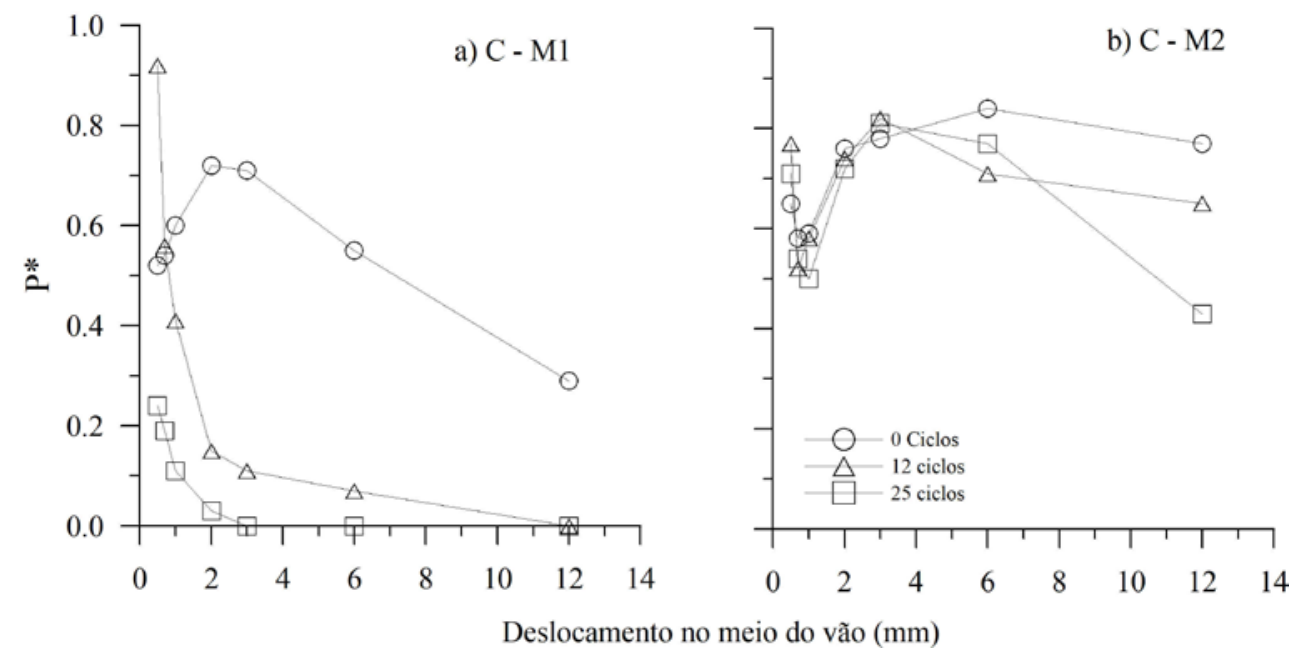

Figura 11 - Processo de ruptura

\begin{tabular}{|l}
\hline $\begin{array}{c}\text { (a) Delaminação do laminado } \\
\text { envelhecido após a primeira } \\
\text { fissuração sob flexão }\end{array}$ \\
$\begin{array}{l}\text { (c) Comprimento de } \\
\text { arrancamento de fibras }\end{array}$ \\
\hline
\end{tabular}

\section{Avaliação microestrutural}

Na Figura 12 é observada a superfície externa lateral da fibra de sisal antes de ser utilizada no compósito e após ser retirada do laminado C-M1, submetido a 12 ciclos de envelhecimento. Verificase que, após o envelhecimento, as fibras perdem parte da rugosidade superficial (células do parênquima que recobrem a superfície), enquanto produtos de hidratação se aderem à superfície da fibra.

Para identificar o tipo de material presente sobre a superfície e lúmen das fibras, foram realizadas cinco análises por EDS por fibra para mapeamento dos elementos químicos $\mathrm{Si}$, Ca, $\mathrm{K}$ e $\mathrm{Na}$. O espectro obtido da análise da fibra, em compósito submetido a 12 ciclos de envelhecimento, está apresentado na Figura 13 e indica a presença de carbono e oxigênio, natural de produtos orgânicos, e de íons cálcio e traços de sílica oriundos da matriz de cimento. Resultado similar foi obtido por Savastano, Warden e Coutts (2005) e corrobora a hipótese da mineralização da fibra de sisal devido à migração do cálcio livre para seu interior.

Os espectros EDS das matrizes M1 e M2 e a análise semiquantitativa de seus componentes químicos estão apresentados na Figura 14 e na Tabela 4 respectivamente. Verifica-se que a relação $\mathrm{CaO} / \mathrm{SiO}_{2}$ para a mistura M1 reduziu de 1,18 para 0,44, o que indica redução do teor de cálcio após o envelhecimento. Para a mistura M2, a relação $\mathrm{CaO} / \mathrm{SiO}_{2}$ manteve-se praticamente constante após 12 ciclos de envelhecimento. Tal comportamento deve-se ao fato de o cálcio presente na mistura contendo metacaolinita fazer parte dos silicatos de cálcio hidratados, que são produtos menos solúveis que o hidróxido de cálcio.

Para efeito de comparação, verifica-se que para a mistura M1 o teor de óxido de alumínio, que é um composto menos solúvel, variou pouco com o envelhecimento acelerado. A relação $\mathrm{Al}_{2} \mathrm{O}_{3} / \mathrm{SiO}_{2}$ aumentou de 0,29 para 0,35. Para a mistura M2, a relação $\mathrm{Al}_{2} \mathrm{O}_{3} / \mathrm{SiO}_{2}$ aumentou de 0,65 para 0,68 . 
Figura 12 - Superfície da fibra de sisal antes do uso e após envelhecimento do compósito
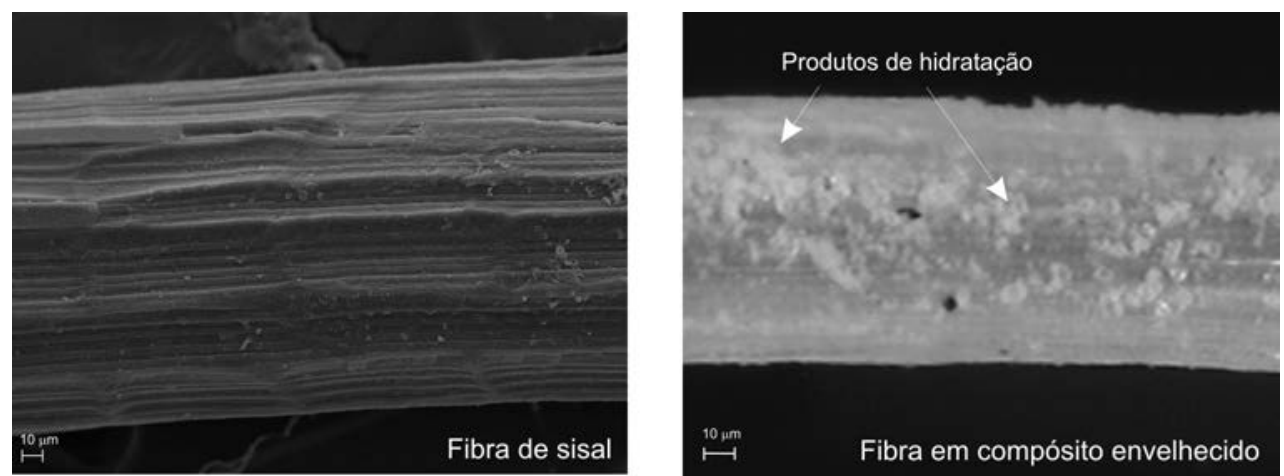

Figura 13 - Imagem de elétrons retroespalhados de superfície de fibra em matriz M1 após 12 ciclos de envelhecimento

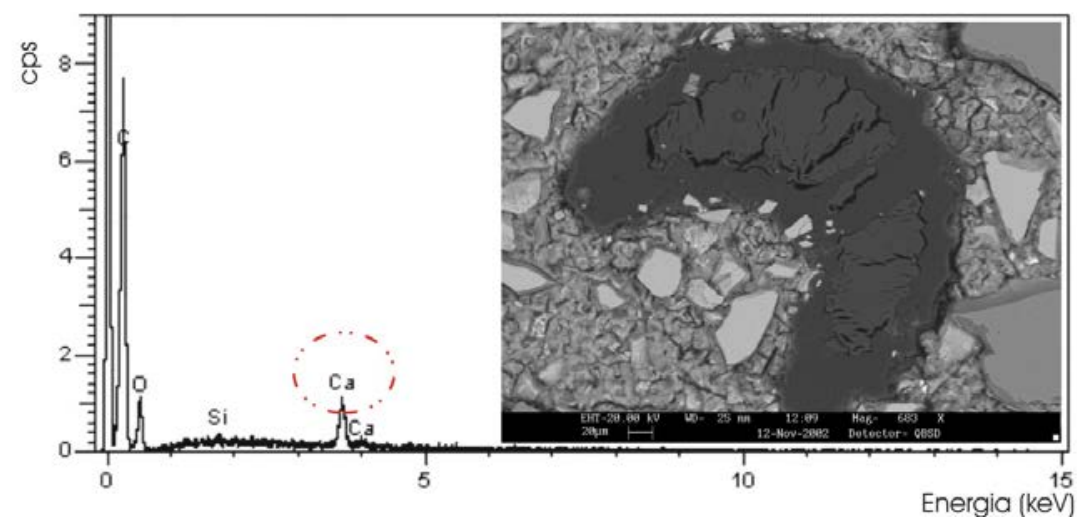

Figura 14 - Imagem de elétrons retroespalhados de superfície de fibra em matriz M1 e M2 sem envelhecimento ( 0 ciclos)
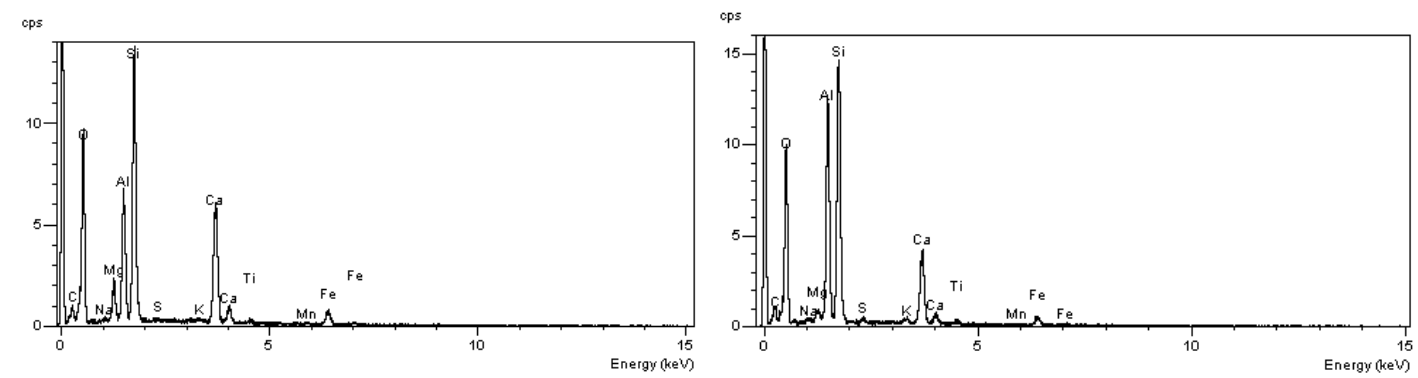

Tabela 4 - Composição química, em porcentagem, de pasta de cimento dos microconcretos obtida através de análise semiquantitativa por EDS

\begin{tabular}{c|c|c|c|c}
\hline Composto & \multicolumn{2}{|c|}{ M1 $^{*}$} & \multicolumn{2}{c}{ M2 } \\
\hline & 0 ciclos & 12 ciclos & 0 ciclos & 12 ciclos \\
\hline $\mathrm{Al}_{2} \mathrm{O}_{3}$ & $9,8(11,2)$ & $16,7(10,2)$ & $29,0(1,1)$ & $29,4(4,3)$ \\
$\mathrm{SiO}_{2}$ & $33,5(2,7)$ & $47,2(3,8)$ & $44,7(8,5)$ & $43,5(2,8)$ \\
$\mathrm{CaO}$ & $39,7(6,3)$ & $21,0(14,9)$ & $17,6(24,9)$ & $17,3(16,3)$ \\
\hline
\end{tabular}

Nota: *Coeficiente de variação entre parênteses, em \% 


\section{Conclusão}

A utilização de adições minerais como a metacaolinita em substituição a parte do cimento pode resultar em uma pasta cimentícia isenta de hidróxido de cálcio livre. Para o cimento e metacaolinita utilizado neste trabalho, foi verificado através de análise termogravimétrica que teores de substituição de $30 \%$ e $40 \%$, em massa, resultam em uma pasta sem teores de $\mathrm{CH}$ mesmo após 730 dias de hidratação. Para pasta com 20\% de substituição ainda é possível verificar a presença de CH após 28 dias de hidratação.

A vantagem da isenção de $\mathrm{CH}$ é a proteção da fibra de sisal contra o processo de mineralização, causado pela migração desse íon para o interior da fibra. De fato, para os laminados produzidos com concretos sem adição mineral, cujo teor de $\mathrm{CH}$ na pasta é da ordem de 7\%, foi verificada total redução da capacidade portante após o surgimento da primeira fissura, após a realização do envelhecimento acelerado. Isso porque as fibras apresentaram ruptura frágil e não conseguiram transferir as tensões. Análises por MEV e EDS das fibras imersas na matriz sem adição comprovaram a migração de íons Si e Ca para a superfície da fibra, o que comprova a teoria da mineralização da fibra pelo $\mathrm{CH}$. De fato, a análise semiquantitativa por EDS da matriz sem adição comprovou a redução do teor de cálcio após o envelhecimento.

Para laminados produzidos com adição de metacaolinita, por outro lado, verificou-se a estabilidade química da pasta, sem variação no teor de cálcio após o envelhecimento, e manutenção das propriedades mecânicas de flexão. A avaliação da superfície de fratura dos laminados demonstra que as fibras mantiveram comprimento de arrancamento similar ao dos laminados sem envelhecimento.

Apesar da manutenção da integridade da fibra, verifica-se, no entanto, que a adição da metacaolinita no microconcreto resulta em um material com menor tensão de fissuração e menos resistente à variação termo-hídrica. Após o envelhecimento acelerado foi verificada intensa microfissuração gerada pelos ciclos de choque térmico, assim como o surgimento de fissuras transversais na face superior e na face inferior do laminado, e de fissuras longitudinais na lateral, ao longo da camada de fibra.

Isso indica que, além do estabelecimento de medidas de proteção à fibra de sisal, é preciso projetar e dosar os microconcretos com o objetivo de garantir uma melhor compatibilidade físicoquímica com as fibras, de forma a evitar o surgimento de fissuras devido a variações bruscas de umidade ou temperatura.

\section{Referências}

BARROS, J. A. O.; SILVA, F. A.; TOLEDO

FILHO, R. D. Experimental and Numerical

Research on the Potentialities of Layered Reinforcement Configuration of Continuous Sisal Fibers For Thin Mortar Panels. Construction and Building Materials, v. 102, p. 792-801, 2016.

BERGSTRÖM, S. G.; GRAM, H. E. Durability of Alkali-Sensitive Fibres in Concrete. International Journal of Cement Composites and Lightweight Concrete, v. 6, n. 2, p. 75-80, 1984.

CLARAMUNT, J. et al. Natural Fiber Nonwoven Reinforced Cement Composites as Sustainable Materials For Building Envelopes. Construction and Building Materials, v. 115, p. 230-239, 2016.

COURARD, L. et al. Durability of Mortars Modified With Metakaolin. Cement and Concrete Research, v. 33, p. 1473-1479, 2003.

DINAKAR, P.; SAHOO, P. K.; SRIRAM, G. Effect of Metakaolin Content on the Properties of High Strength Concrete. International Journal of Concrete Structures and Materials, v. 7, n. 3, p. 215-223, 2013.

DWECK, J. et al. Hydration of a Portland Cement Blended With Calcium Carbonate.

Thermochimica Acta, v. 346, n. 1, p. 105-113, 2000.

FERREIRA, S. R. et al. Effect of Fiber Treatments on the Sisal Fiber Properties and Fiber-Matrix Bond in cement Based Systems. Construction and Building Materials, v. 101, p. 730-740, 2015.

GRAM, H. E. Durability of natural fibres in concrete. Swedish Cement and Concrete Research Institute, v. 1, n. 83, 1983.

INSTITUTE BELGE DE NORMALISATION. NBN B 15 238: essais des betons reforce de fibres: essai de flexion sur éprouvettes prismatiques. Brussels, 1992.

LIMA, P. R. L. Análise Teórica e Experimental de Compósitos Reforçados Com Fibras de Sisal. Rio de Janeiro, 2004. Thesis (PhD) - COPPE, Universidade Federal do Rio de Janeiro, Rio de Janeiro, 2004.

LIMA, P. R. L.; TOLEDO FILHO, R. D. Uso de metacaulinita para incremento da durabilidade de Compósitos à Base de Cimento Reforçados Com Fibras de Sisal. Ambiente Construído, Porto Alegre, v. 8, n. 4, p. 7-19, 2008. 
LIMA, P. R. L. et al. Characterization and Treatment of Sisal Fibers From Agricultural Residues for Use in Cement-Based Composite. Engenharia Agrícola, v. 34, n. 5, p. 812-825, 2014.

LIMA, P. R. L. et al. Potentialities of CementBased Recycled Materials Reinforced With Sisal Fibers as a Filler Component of Precast Concrete slabs. In: SAVASTANO, H.; FIORELLI, J.; SANTOS, S. F. (Eds.). Sustainable and Nonconventional Construction Materials Using Inorganic Bonded Fiber Composites. Elsevier, 2017a.

LIMA, P. R. et al. Effect of Surface Biopolymeric Treatment on Sisal Fiber Properties and FiberCement Bond. Journal of Engineered Fabrics \& Fibers, v. 12, n. 2, 2017b.

MOHR, B. J.; BIERNACKI, J. J.; KURTIS, K. E. Supplementary Cementitious Materials For Mitigating Degradation of Kraft Pulp FiberCement Composites. Cement and Concrete Research, v. 37, n. 11, p. 1531-1543, 2007.

MOTA, J. M. F.; OLIVEIRA, R. A.; CARNEIRO A. M. Durabilidade de Argamassas Com Adição de Metacaulim Para Reforço de Alvena. Revista Matéria, v. 21, n. 4, p. 1105-1116, 2016.

PAPADAKIS, V. G.; FARDIS, M. N.;

VAYENAS, C. G. Hydration and Carbonation of Pozzolanic Cements. Materials Journal, v. 89, n. 2, p. 119-130, 1992.

ROMA, L. C.; MARTELLO, L. S.;

SAVASTANO, H. Evaluation of Mechanical, Physical and Thermal Performance of CementBased Tiles Reinforced With Vegetable Fibers. Construction and Building Materials, v. 22, n. 4, p. 668-674, 2008.
SAVASTANO, H.; WARDEN, P. G.; COUTTS, R. S. P. Microstructure and Mechanical Properties of Waste Fibre-Cement Composites. Cement and Concrete Composites, v. 27, n. 5, p. 583-592, 2005.

SILVA, A. S. et al. Long-Term Behavior of limeMetakaolin Pastes at Ambient Temperature and Humid Curing Condition. Applied Clay Science, v. 88/89, p. 49-55, 2014.

TOLEDO FILHO, R. D. et al. Durability of Alkali-Sensitive Sisal and Coconut Fibres in Cement Mortar Composites. Cement and Concrete composites, v. 22, n. 2, p. 127-143, 2000.

TOLEDO FILHO, R. D. et al. Development of Vegetable Fibre-Mortar Composites of Improved Durability. Cement and Concrete Composites, v. 25, n. 2, p. 185-196, 2003.

TONOLI, G. H. D. et al. Effect of Accelerated Carbonation on Cementitious Roofing Tiles Reinforced With Lignocellulosic Fibre.

Construction and Building Materials, v. 24, n. 2, p. 193-201, 2010.

WEI, J.; MEYER, C. Degradation Mechanisms of Natural Fiber in the Matrix of Cement Composites. Cement and Concrete Research, v. 73, p. 1-16, 2015.

WILD, S.; KHATIB, J. M.; JONES, A. Relative Strength, Pozzolanic Activity and Cement Hydration in Superplasticised Metakaolin Concrete. Cement and Concrete Research, v. 26, n. 10, p. 1537-1544, 1996. 


\section{Paulo Roberto Lopes Lima}

Departamento de Tecnologia | Universidade Estadual de Feira de Santana | Av. Transnordestina, s/n, Novo Horizonte | Feira de Santana BA - Brasil | CEP 44036-900 | Tel.: (75) 3161-8310 | E-mail: lima.prl.uefs@gmail.com

\section{Romildo Dias Toledo Filho}

Programa de Engenharia Civil | Universidade Federal do Rio de J aneiro | Cidade Universitária, Bloco B, Ilha do Fundão | Rio de J aneiro RJ - Brasil | CEP 21945-970 | Tel.: (21) 2562-8479 | E-mail: toledo@coc.ufrj.br

\section{Reiner Neumann}

Setor de Caracterização Tecnológica, Coordenação de Análises Minerais | Centro de Tecnologia Mineral | Av. Pedro Calmon, 900, Cidade Universitária | Rio de J aneiro - RJ - Brasil | CEP 21941-908 | Tel.: (21) 3865-7263 | E-mail: rneumann@cetem.gov.br

\section{J oaquim Antonio Oliveira Barros}

Departamento de Engenharia Civil | Universidade do Minho | Campus Azurém | Guimarães - Portugal | E-mail: barros@civil.uminho.pt

\section{Revista Ambiente Construído}

Associação Nacional de Tecnologia do Ambiente Construído

Av. Osvaldo Aranha, 99 - 30 andar, Centro

Porto Alegre - RS - Brasil

CEP $90035-190$

Telefone: +55 (51) 3308-4084

Fax: +55 (51) 3308-4054

www. seer. ufrgs. br/ ambienteconstruido

E-mail: ambienteconstruido@ufrgs.br 